\title{
Mobility-based routing algorithm in delay tolerant networks
}

\author{
Marcin Kawecki and Radosław Olgierd Schoeneich
}

\begin{abstract}
The paper presents a routing algorithm based on the use of the mobility of the nodes in the Delay and Disruptive Tolerant Networks (DTN). DTN are characterized by temporary or permanent lack of a continuous path between the source and the destination node. The communication is done by ferrying the message by intermediate nodes based on store-carry-forward paradigm. Our routing algorithm is based on the ability to use information about node mobility and their contacts. We assume that the greater mobility of node results in higher number of contacts with other nodes and higher probability in message delivery to the destination. The proposed algorithm was simulated using The One simulation tool. We compare the performance of our algorithm and other popular routing algorithms.
\end{abstract}

Keywords: Disruption tolerant networking, Mobile ad hoc networks, Mobile communication, Routing protocols, Wireless networks

\section{Introduction}

Internet network over the past several years achieved a huge success in the communication between devices. This has become possible thanks to the use of uniform sets of protocols by all users connected in the networks. All the devices connected to the Internet use protocols which belong to the TCP/IP protocol stack. Internet communication is based on several assumptions. Basic is the existence of a continuous, two-way path connecting the source and a destination node in order to allow endto-end communication. Despite the great success of these networks, they cannot be adapted to any environment due to the cost associated with the necessary infrastructure, as well as the specifics of the environment which can be characterized by the long delays, interruptions in the transmissions, limited buffer, and energy resources of nodes.

Delay and Disruptive Tolerant Networks (DTN) can be characterized by the lack of the possibility of establishing a path between any pair of nodes at the time. In the DTN, communication uses store-carry-and-forward paradigm. This is done by using nodes physically carrying messages called message ferries or message mules.

\footnotetext{
* Correspondence: M.Kawecki@stud.elka.pw.edu.pl

Institute of Telecommunications, Warsaw University of Technology, Warsaw, Poland
}

\section{State of the art}

Works on wireless DTN are conducted intensively, especially focus on routing protocols.

One of the basic and most popular routing algorithms in DTN is Epidemic routing (ER) [6]. The algorithm operates on the principle of "flooding" network. The Epidemic routing node that received the message forwards it to all nodes encountered by him. The message is thus propagated through the network, so that all nodes have

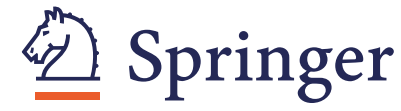

DTN provide the ability to communicate in specific scenarios. Examples of practical applications are provision of communication in rural areas in India and other regions Where there are no fixed network infrastructure [1] warning drivers about the current traffic situations, projects for the observation of wild animals [3], monitoring of the pollution of water pools or road noise [4], or maintaining communications through interThis paper presents the idea of using the mobility of carriers and contacts between mobile devices for wireless messaging. In the following sections will be presented sequentially the state of art (Chapter 2), a routing protocol design using mobility property (Chapter 3 ), and the results of simulation studies (Chapter 4). The work was summarized in Chapter 5. 
a copy of the sent message by the source node. This means that each message is stored in the node's local cache. Epidemic routing algorithm provides a messaging with the minimum possible delay [7], due to the fact that there are many paths used to deliver the message. The main disadvantage of Epidemic routing algorithm is requiring a large amount of resources such as buffer capacity, bandwidth, and energy resources of nodes.

Another very popular protocol in DTN is Spray and Wait [8]. The solution allows for far less number of transmissions compared to the Epidemic routing and is faster and more efficient than the algorithms based on the transfer of one or more copies of messages in the network. In the Spray and Wait, there can be distinguished two phases: spray-during which the message is replicated and wait-during which a node does not transmit a message, but it is trying to deliver a message to the destination node in the direct contact.

Another routing algorithm is the Prophet [9]. It is an algorithm based on the assumption of non-random movement of nodes. It is also assumed that the nodes appear in some locations more often than others, and therefore the appearance of node in the visited locations in the past is more likely to occur in the future. Nodes maintain the probability of meeting the other nodes in the network and based on this information they communicate with more appropriate nodes, which have higher probability value.

In many networks, the main carriers of messages are people. The characteristics of community created between people can be helpful in improving the transmission of messages. In [10], the authors propose a labeling strategy to choose which node should forward the message. People belonging to the same community come together often and are suitable carriers for messages destined for their community. Each node has assigned a label belonging to the group. Nodes select other nodes that belong to the same community based on label. Unfortunately, it can lead to increased delay in transmission when a node for a long time did not meet another node belonging to the same community.

In [11], the authors propose an algorithm SimBet. It uses centrality and the similarity metrics to choose relay node, for each node in a local neighborhood is made centrality estimation. Similarity metric is calculated from the group of neighbors of a node and the destination node. Nodes that have a high centrality are treated as a kind of "bridges" in its neighborhood, and the nodes of high similarity with the target node are potentially good neighbor nodes to find the destination node.
Another algorithm that uses a centrality is the Bubble Rap [12]. The authors assume that each node belongs to at least one community, and its centrality describes the popularity of a given node in his community. Each node has a global centrality metric across the network and the local on its local community. Using the described social characteristics in Bubble Rap, there can be distinguished two phases: the transmission of messages based on global centrality and transmission of messages based on local centrality. In both phases, the messages are sent to the nodes with a higher popularity (centrality). When the node has a message for the destination node, first, it passes the message based on global centrality by the time the message will be in the same community as the destination node. At this point, the algorithm proceeds to the second phase of forwarding the message based on the local popularity. The authors [13] present a work based on existing human relationships. People usually make friends with other people if they share common interests and activities, thus meet more often. Spreading a similar type of data takes place between friends, and other types of data are transmitted to the foreign nodes. Diffusion of data with a similar profile takes place when a pair of nodes friends met, because they represent a common interest. A pair of stranger nodes exchange data unrelated to their interest. In [14], authors proposed the use of friendship between the nodes to transmit packets in DTN. Friendship between nodes is characterized on the basis of the frequency of contacts and number of long-lasting, regular contacts. To determine the quality of friendship, Social Pressures Metric (SPM) is used. The value of this metric between a pair of nodes can be determined based on the history of contacts between the nodes. SPM metric can help in appropriate relaying messages, but its calculation requires information on all your contacts during each period, which may not be appropriate in realistic scenarios. In addition, proposed by authors routing method is similar to the Routing Label [10], which can result in similar problems. If the source node does not meet the node belonging to the same community as the destination node, message will not be delivered. In [15], authors presented an algorithm social-aware and stateless routing (SANE). This strategy uses a profile of interest routing of node in a kdimensional vector Iu. To express the similarity of interests between a pair of nodes, authors introduced cosine similarity metric. In the algorithm, SANE message is relayed to the nodes that have similar interest profiles as the destination node. The advantage of this method is that each node only needs to maintain its own profile interests. The cost of maintaining and 
updating social metrics is relatively low. This solution is highly scalable. The authors of [16] propose a method that uses the centrality and interest of users. Centrality is characterized by the expected number of nodes involved in data belonging to node "i" which node " $\mathrm{i}$ " may encounter during the time period of data spreading. Intermediate nodes are selected so they have better possibility to spread the data to the nodes interested in them than the current node based on the newly defined, time-dependent centrality metric. There are taken into account the local and global-multi-hop centrality. With multi-hop centrality, there are more chances to pass the message, and there is a more accurate estimation of the probability of transfer. In [17], the authors present social-based DTN routing. The approach is based on the idea according to which nodes are more sociable when they often meet different other nodes, as the result, they are good candidates to give them the message. The authors defined a metric sociability indicator to describe the possibility of passing messages through the node. This metric describes the social behavior of a node by counting the number of nodes encountered by this node. Routing strategy is to relay messages to the most social nodes. In the social-based algorithms, there are solutions that use selfish nodes. Selfish node is a node that maximizes its own profits and limits the use of its own resources. The existence of such a large number of nodes in the network may have a negative effect on its performance. Give2get algorithm [18] is based on the reputation of nodes in the network. Information about the source and destination nodes is hidden in the message. After the stage of the negotiations with a potential relay node, the message is sent, but the sending node is expecting to receive a confirmation of further relay of this message. If the confirmation will not be sent back to the source node, the source node sends a broadcast message to all nodes in the network with the information that this node does not transmit a message and, therefore, is excluded from the messaging.

Other important works are focused on scheduling in vehicular DTN [19], multi-cast solution [20, 21]. Many algorithms are based on green technology assumptions for ad hoc networks [22, 23]. Other works are based on multilayer clusters [24], routing quality games [25], spatial reusability [26], and other solutions can be found in [27-44], and complex surveys in works [45, 46].

At the best of our knowledge, none of this works are similar to our algorithm.

\section{Routing algorithm using mobility of nodes}

The main objective of DTN routing algorithm is to obtain a high rate of delivered messages, while maintaining low overhead. Algorithms such as Epidemic Routing, MaxProp, or Prophet can reach a high percentage of delivered messages but at the expense of a very large number of sent messages that cause high overhead. Algorithms such as Spray and Wait and Spray and Focus enable the reduction of overhead messages but do not reach such a high probability of delivery.

Presented in this work replication algorithm achieves high probability of message delivery while maintaining low overhead. The improvement introduced in this algorithm is based on noticing and using the properties of the network whereby the greater mobility of nodes is associated with a higher probability of meeting another node, faster network coverage expansion, which results in better propagation of transmitted messages. This feature can involve many different networks; it appears to be particularly important in an urban environment, where multiple nodes move: people who are owners of communicating with each other, smartphones, and motorized vehicles such as cars, buses, and trams equipped with the appropriate communication interfaces.

Human activity during the day is rather schematic; the man gets up in the morning, at a specified time, and leaves the house, and he goes to work or school by foot, car, or using public transport. Then, usually a person spends at work a certain amount of time, then returns home by chosen way of transport. In the evening, people often go shopping, go out to meet friends, enjoy the entertainment, or remain in their homes. Described scheme of activity in the majority of cases can be assigned to a large part of society. It is repeated periodically, every day. It should be noted that this can contribute to the prediction of human activity in the daytime. To determine the level of activity, there is a need to specify how much mobile node is in a certain period of time. It has been assumed that the nodes in the network are aware of their speed. To determine the speed of a node in a given time interval, GPS can be used. Another solution may be an application that recognizes human activity using a smartphone worn in the pocket.

As mentioned earlier, the presented algorithm uses replication. It limits the number of messages in the network, so that it becomes possible to minimize the use of network resources. Routing algorithm makes decisions about relaying messages to the other nodes based on the mobility, sending a message to the nodes that will show greater mobility in the near future.

Information about the mobility of a node is the local metric of each node. It is calculated as a weighted moving average (WMA) with instantaneous speed node samples taken from a rate of $1 \mathrm{~min}$ according to the formula (1). 


$$
\mathrm{WMA}=\frac{n p_{0}+(n-1) p_{1}+\ldots+p_{n-1}}{n+(n-1)+\ldots+2+1}
$$

WMA assigns different weights to the data from individual periods. In the formula, $p 0$ is the last value which assigned weight is $n$.

The weighted moving average is calculated for each hour of the movement in the day separately, so you can get a level of mobility of a movement at different times of the day, which can change over the day time.

Each node depending on its current and future mobility has assigned one of three mobility class: (a) the first class: the least mobile nodes, e.g., pedestrians; (b) the second class: nodes having an average mobility, for example cyclists, people running; (c) the third class: the most mobile nodes, e.g., moving cars, buses.

The nodes are not statically assigned to the mobility class; depending on changes in its mobility in certain periods of the day, assigned class may change.

\subsection{Algorithm}

Each node, as already mentioned, maintains a local metric of its mobility on the basis of which it is assigned an appropriate mobility class. The assumption of the algorithm is that less mobile nodes transmit their messages to more mobile nodes at a time when it cannot send messages directly to the destination node. When two nodes meet each other, the node that has a message to send checks if this message has the number of replicas greater than 1 . If this condition is met, there are two possible cases: (1) If a node wishing to send a message belongs to the first mobility class and he encountered a node that belongs to a higher class, then he transfer its message and assign this message half the number of replicas. The source node also lowers the number of replicas of the message by half. Otherwise, the message is not passed to encountered node. (2) If a node wishing to send a message not belongs to the first mobility class and encountered node belongs to the same or higher class than the source node mobility class then he transfer its message and assign this message half the number of replicas. The source node also lowers the number of replicas of the message by half. Otherwise, the message is not passed to encountered node.

Depending on the number of replicas initially assigned to the message, a node may be able to transmit the message several times, each time assigning half the current number of replicas to the message. At some point, there may be a situation in which a given node has only one replica assigned to given message. From that moment, when transmitting messages in addition to the node mobility class, encountered nodes in the past are taken into account. The idea is to choose relay node that encountered destination node of the message. Each node keeps the information about which nodes met within the past time. If the time elapsed since the last meeting a pair of nodes is longer than the length of the time window, information about their encounter is lost. In this way, the nodes maintain information about the meeting of nodes with only the immediate past. If at some point there is a meeting of two nodes and the source node has a message which is assigned only one replica, it checks if it was already sent having only one replica. If the message was already sent, no action is taken on. However, if the message has not been sent yet, following situations are possible: (1) If a node wishing to send a message belongs to the first mobility class, and encountered node belongs to a higher class and node encountered met destination node of the message in the near past, then the message is transferred to the encountered node. Otherwise, the message is not passed to encountered node. (2) If a node wishing to send a message does not belong to the first mobility class and the encountered node met destination node of the message in the near past, then the message is transferred to encountered node. Otherwise, the message is not passed to encountered node.

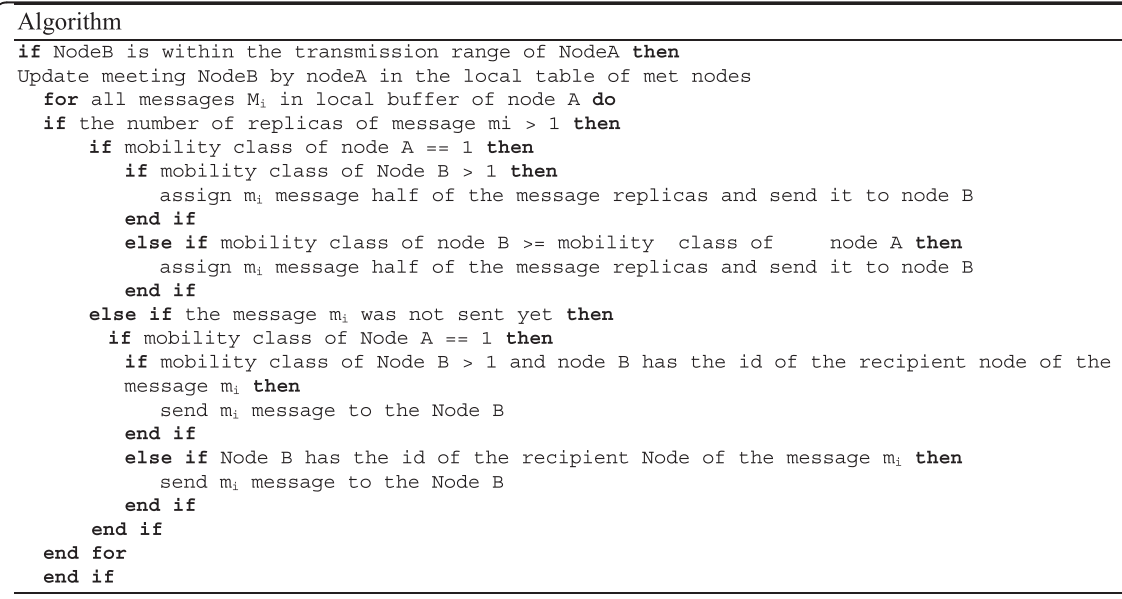




\section{Simulations}

In this chapter, we analyze and compare the performance of the proposed algorithm with three popular routing algorithms: Epidemic [6], Spray and Wait [8], and the Prophet [9]. All simulations were performed in the simulator The ONE [47]. At the beginning, it will be presented mobility model and parameters used for the simulation, then the simulation results.

\subsection{Mobility model}

In the performed simulations, we used a city map of Helsinki about the size of $10,000 \times 8000 \mathrm{~m}^{2}$. In order to increase the realism of the simulation, we selected Working Day Movement (WDM) [48] mobility model. Simple models such as Random Walk, Random Waypoint, or more complex Random Map Based Movement does not reflect such characteristics as the relationship between the carriers which may be people or their repeated behavior. Working Model Day Movement makes the movement of nodes even more realistic. In this model, the movement assumes that people-carriers move in accordance with certain routines, e.g., walking to work, shopping. Thanks to this node which often meets with a specific group. The node is not able to meet on their way all nodes due to its motion introduced locality. Workplace, position of the house in which he resides makes that node movement, is often restricted to visiting certain locations.

WDM model divides its simulation time for days; within every day, there are three node following activities: (1) home activity, (2) work-related activity, and (3) activity related to entertainment

These three activities can be varied and can have individual character for different people. However, these are activities that can be attributed to the typical day of a large number of people.

\subsection{Simulation parameters}

The number of nodes during the simulation is fixed at 500 nodes. Nodes are deployed in seven districts of the city, where there are their homes, workplaces, and places associated with entertainment. Parts of nodes are the buses, which are divided into bus lines-groups. Each district has its own bus line, from which can benefit nodes belonging to this district. Primary nodes are moving at a speed of $0.8-1.7 \mathrm{~m} / \mathrm{s}$, and the nodes being buses with values of between 5 and $10 \mathrm{~m} / \mathrm{s}$. Buses stop at bus stops for a period of time between 10 and $30 \mathrm{~s}$. Nearly half of the nodes may have a car to ride around the city (Table 1 ).

The behavior of the nodes has been further diversified by setting up different hours of the morning node's waking up. Nodes leave their homes between the 5 and 12 . The working time was set at $28,800 \mathrm{~s}(8 \mathrm{~h})$. Break time
Table 1 Simulation parameters

\begin{tabular}{ll}
\hline Parameters & Description \\
\hline Simulation area & $10,000 \mathrm{~m} \times 8000 \mathrm{~m}$ \\
Number of nodes & 500 \\
Radio range & $10 \mathrm{~m}, 100 \mathrm{~m}$ \\
Buffer space & $1 \mathrm{Mb}, 50 \mathrm{Mb}$ \\
Packet payload & $1-300 \mathrm{~Kb}$ \\
TTL & $24 \mathrm{~h}$ \\
\hline
\end{tabular}

in office has the Pareto distribution, a factor of 0.5 and its minimum value is $10 \mathrm{~s}$. Office is the area of the square size of $100 \times 100 \mathrm{~m}$. Each node may perform the evening activity with a probability equal to 0.5 ; the size of the groups during the evening activity is from one to three nodes. The nodes are equipped with a Bluetooth radio interface. Buses also have high speed radio interfaces $10 \mathrm{Mbit} / \mathrm{s}$ and $100 \mathrm{~m}$ range.

\subsection{Simulation results}

Each simulation is repeated several times to achieve more accurate and independent from the initial position of nodes results. The simulations are performed with different grain generator, and the same configuration is repeated for different routing algorithms. The results are estimated with the $95 \%$ confidence intervals. Comparing the effectiveness of simulated algorithms is taken into account the following criteria: probability of message delivery, efficiency, overhead ratio, number of hops, and average delay.

Figure 1 shows the dependence of the probability of delivery of messages to the length of simulation time. For all algorithms with time increase, the probability of message delivery also increases, the maximum value is reached after a simulated 4 days. After the first day of the simulation, all algorithms obtain similar results, which change over time. In the case of the Epidemic routing, the lowest probability is probably connected with buffer overflows as a result of the transmission of the messages to all nodes encountered, causing rejecting new messages. The proposed algorithm and the Prophet exceed a threshold of about $80 \%$ delivered messages after 4 days of simulation.

Overhead ratio is interpreted as the number of relayed messages needed to deliver single message to the destination node. The results for the overhead ratio of different algorithms are shown in Fig. 2. Algorithms Epidemic routing and Prophet have very high overhead ratio, many times larger than the proposed algorithm and Spray and Wait (logarithmic scale on the graph). The proposed algorithm achieves the best result among all algorithms. Overhead ratio of proposed algorithm is lower than the Spray and Wait, which is also limiting the number of transmissions. The number of replicas 


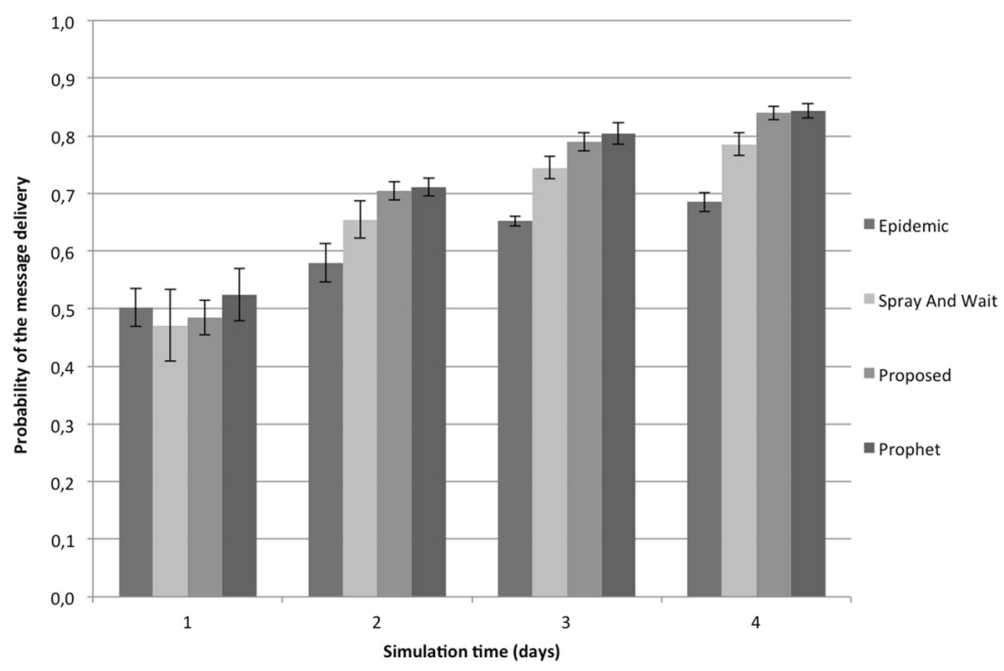

Fig. 1 Probability of message delivery

assigned to each created message in the Spray and Wait in the simulation is 32 . The proposed algorithm assigns message 16 replicas for nodes belonging to the first mobility class, 8 replicas for nodes belonging to the second class, and one for the third class.

Number of messages sent by the nodes in the network can have a big impact on the probability of message delivery. Unfortunately, a large number of messages sent is associated with greater overhead ratio, these two metrics are dependent on each other. It would be good to know how messages that are sent by the nodes affect the number of delivered messages. Therefore, we introduced a new metric defined as the efficiency of the algorithm. Efficiency is the ratio of the number of the messages delivered to the number of all transmissions of the messages. The results of the efficiency of the tested algorithms are shown in Fig. 3. As the graph shows, the proposed algorithm achieves the highest degree of efficiency. A high value has also Spray and Wait. The lowest value has Epidemic routing because of the very large number of transmissions. Prophet algorithm, which reached a very high probability of message delivery, also due to the large number of transmissions has a very low efficiency

Average delay is the average time that elapses from the creation of message until its delivery. The simulation results of the average delay of delivery of messages are shown in Fig. 4. As expected, the lowest value achieves Epidemic routing, thanks to the fact that a message is quickly and repeatedly copied on the network, resulting

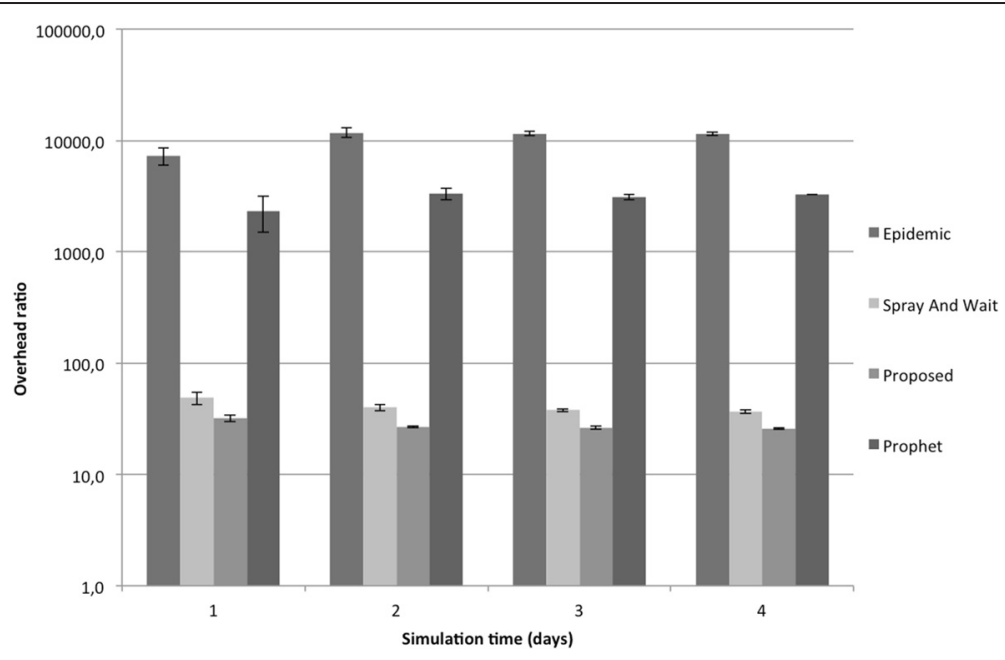

Fig. 2 Overhead ratio 


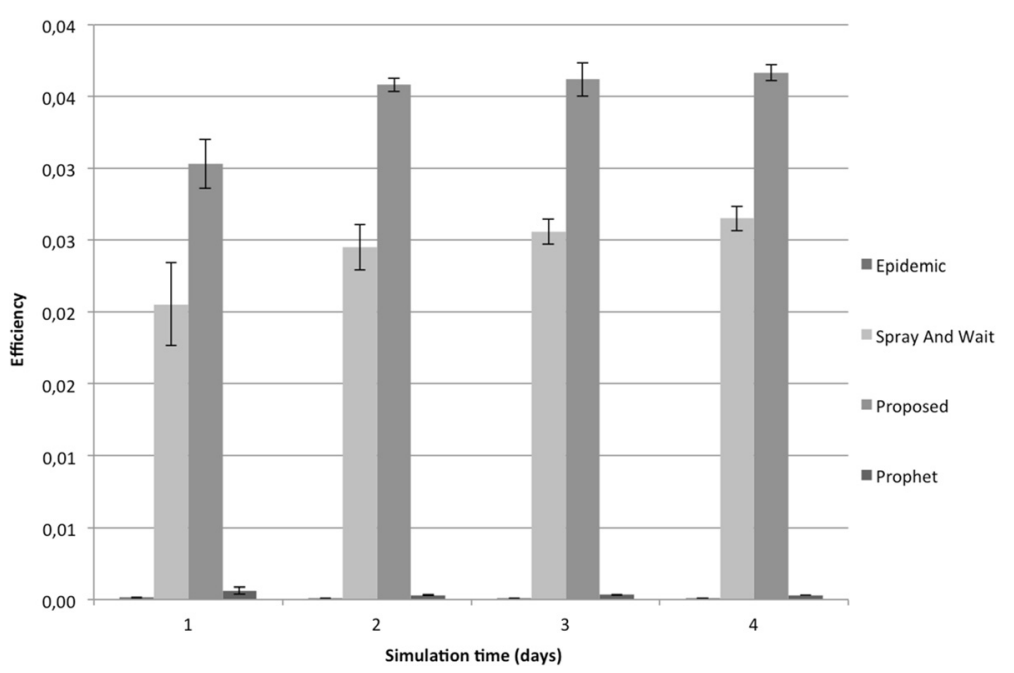

Fig. 3 Efficiency

in greater opportunity to reach the destination node. Prophet gets higher than Epidemic delay but lower than Spray and Wait, and the proposed algorithm. A high average delay of Spray and Wait and the proposed algorithmic likely due to their strong reducing the amount of network transmissions, so the message is relayed by a smaller number of nodes, extending the time of delivery to the destination node.

Hop count metric determines the number of hops from the source and the destination node. This represents also transmission cost, telling how many nodes are on average involved in delivering the message. Results of the simulation are shown in Fig. 5. The highest number of hops has Epidemic routing, due to copying messages to all nodes encountered. A large number of the transmissions cause the Prophet algorithm to have a high value of hop count metric, but lower than Epidemic routing. Spray and Wait and the proposed algorithm have very similar, low value of the number of hops. It is dependent on the initial number of replicas assigned to the newly created message.

\section{Conclusions}

Proper and efficient data transfer is a key issue for DTN. Existing algorithms based on the "flooding" technique can reach a high probability of message delivery. However, a large number of sent messages make over-used network resources such as bandwidth, or storage. Excessive use of resources can lead to overloading and thus rejecting packets. In this work, we show that by sending a small number of messages addressed to the selected nodes, you can get good results in social DTN. The

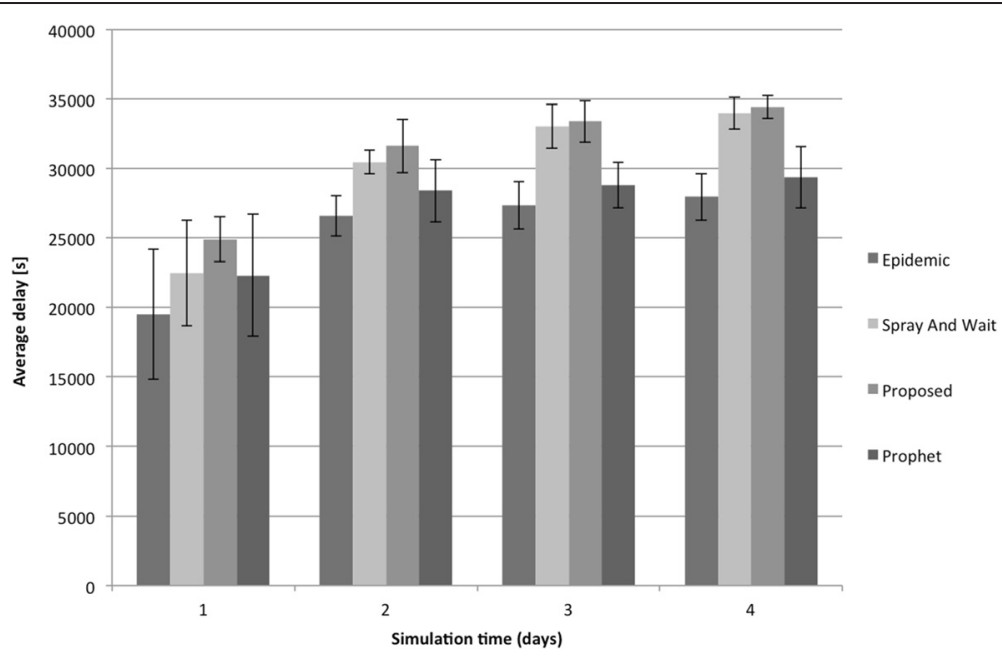

Fig. 4 Average delay 


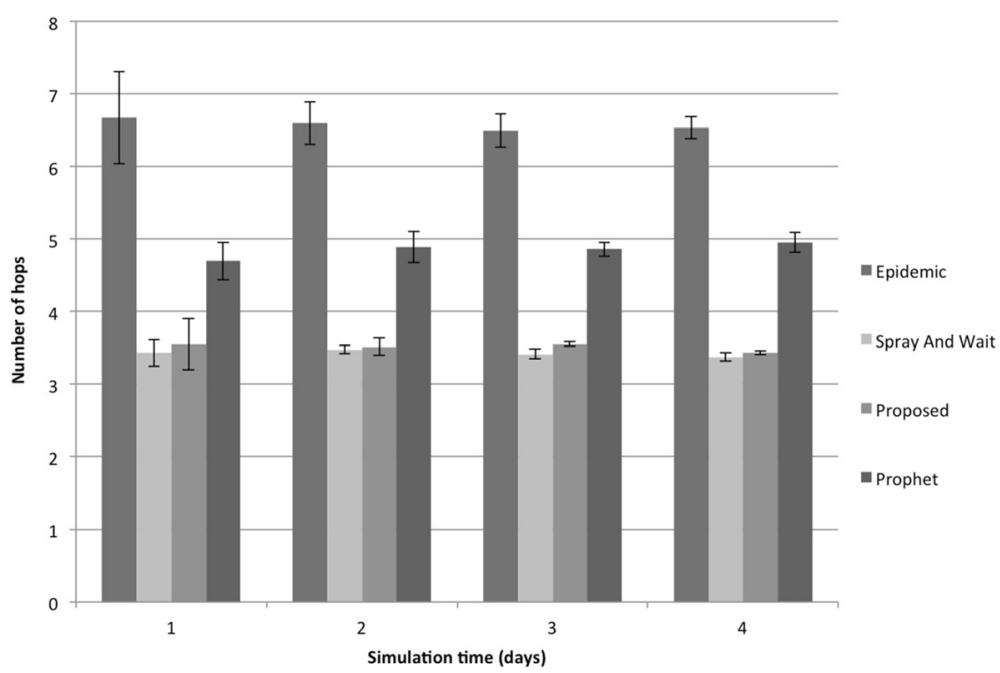

Fig. 5 Average hop count

choice of nodes in the proposed algorithm is based on the characteristics of mobility of nodes and contacts between nodes. Algorithm selects nodes, which have a higher mobility, thus increasing the probability of encountering greater number of nodes, including the destination node of the message. Another factor to consider when transmitting messages is contacts between nodes. In the second phase of the algorithm, there are selected nodes, which have been in contact with the destination node of the message in the recent past. Made simulations showed that the proposed algorithm achieves better results of overhead ratio and effectiveness, with comparable or greater probability of message delivery in relation to other algorithms such as Epidemic routing, representing the technique of "flooding" Spray and Wait, which is an example of limited replication algorithms and the Prophet representing a group of probabilistic algorithms.

\section{Competing interests}

The authors declare that they have no competing interests.

Received: 1 December 2015 Accepted: 2 March 2016

Published online: 11 March 2016

\section{References}

1. A Pentland, R Fletcher, A Hasson, DakNet: rethinking connectivity in developing nations. Computer, IEEE Publishing Society, 78-83 (2004). doi:10.1109/MC.2004.1260729

2. C Maihöfer, R Eberhardt, Time-stable geocast for ad hoc networks and its application with virtual warning signs. Comput. Commun. 27(11), 1065-1075 (2004). doi:10.1016/j.comcom.2004.01.024

3. P Juang, H Oki, Y Wang, M Martonosi, LS Peh, D Rubenstein, Energy-efficient computing for wildlife tracking: design tradeoffs and early experiences with ZebraNet. Proceedings of the 10th international conference on Architectural support for programming languages and operating systems, 2002, pp. 96-107. doi:10.1145/605397.605408

4. P McDonald, D Geraghty, I Humphries, S Farrell, V Cahill, Sensor networking with delay tolerance. Proc. 16th Int. Conf. Comput. Commun. Netw, 2007, pp. 1333-1338. doi:10.1109/ICCCN.2007.4318006
5. S Burleigh, A Hooke, L Torgerson, K Fall, V Cerf, B Durst, K Scott, H Weiss, Delay-tolerant networking: an approach to interplanetary internet. IEEE Commun. Mag., 128-136 (2003). doi:10.1109/MCOM.2003.1204759

6. A Vahdat, D Becker, Epidemic routing for partially connected ad hoc networks. Technical Report, Raport CS- 200006 (Duke University, Durham, 2000)

7. X Zhang, G Neglia, J Kurose, D Towsley, Performance modeling of epidemic routing. Computer Networks: The International Journal of Computer and Telecommunications Networking 51, 2867-2891 (2007). doi:10.1016/j.comnet.2006.11.028

8. T Spyropoulos, K Psounis, CS Raghavendra, Spray and wait: an efficient routing scheme for intermittently connected mobile networks. Proceedings of the 2005 ACM SIGCOMM workshop on Delay-tolerant networking, 2005, pp. 252-259. doi:10.1145/1080139.1080143

9. A Lindgren, A Doria, O Schelén, Probabilistic routing in intermittently connected networks. ACM SIGMOBILE Mobile Computing and Communications Review, 19-20 (2003). doi:10.1145/961268.961272

10. P Hui, J Crowcroft, How small labels create big improvements. International Workshop on Intermittently Connected Mobile Ad hoc Networks in conjunction with IEEE PerCom 2007, 65-70 (2007). doi:10.1109/PERCOMW. 2007.55

11. EM Daly, M Haahr, Social network analysis for routing in disconnected delay-tolerant manets, in MobiHoc '07 Proc. 8th ACM international symposium on Mobile ad hoc networking and computing, 2007, pp. 32-40. doi:10.1145/ 1288107.1288113

12. P Hui, J Crowcroft, E Yoneki, Bubble rap: Social-based forwarding in delay tolerant networks, in Proc. 9th ACM International Symposium on Mobile Ad Hoc Networking and Computing (MobiHoc), 2008, pp. 241-250. doi:10.1109/TMC.2010.246

13. Y Zhang, J Zhao, Social network analysis on data diffusion in delay tolerant networks, in MobiHoc '09: Proc. Tenth ACM International Symposium on Mobile Ad Hoc Networking and Computing, 2009, pp. 345-346. doi:10.1145/1530748.1530806

14. E Bulut, BK Szymanski, Friendship based routing in delay tolerant mobile social networks, in Proc. IEEE Global Telecommunications Conference (GLOBECOM), 2010, pp. 1-5. doi:10.1109/GLOCOM.2010.5683082

15. A Mei, G Morabito, P Santi, J Stefa, Social-aware stateless forwarding in pocket switched networks, in Proc. 30th IEEE Conference on Computer Communications(INFOCOM) mini-conference, 2011, pp. 251-255. doi:10. 1109/TPDS.2014.2307857

16. W Gao, G Cao, User-centric data dissemination in disruption tolerant networks, in Proc. 30th IEEE Conference on Computer Communications (INFOCOM), 2011, pp. 3119-3127. doi:10.1109/INFCOM.2011.5935157

17. F Fabbri, R Verdone, A sociability-based routing scheme for delay-tolerant networks. In EURASIP Journal on Wireless Communications and Networking 2011, 1-13 (2011). doi:10.1155/2011/251408 
18. A Mei, J Stefa, Give2get: forwarding in social mobile wireless networks of selfish individuals, in ICDCS '10 Proc. 2010 IEEE 30th International Conference on Distributed Computing Systems, (2010), pp. 1-13. doi:10.1109/ICDCS.2010.36

19. $\quad Y$ Zeng et al., Directional routing and scheduling for green vehicular delay tolerant networks. Wirel. Netw 19(2), 161-173 (2013)

20. P Li et al., CodePipe "An opportunistic feeding and routing protocol for reliable multicast with pipelined network coding" (INFOCOM, 2012), pp. 100-108. doi: 10.1109/INFCOM.2012.6195456.

21. $P$ Li et al., Reliable multicast with pipelined network coding using opportunistic feeding and routing. IEEE Transactions on Parallel \& Distributed Systems 25(12), 3264-3273 (2014)

22. S Yuning et al., A biology-based algorithm to minimal exposure problem of wireless sensor networks. IEEE Trans. Netw. Serv. Manag. 11(3), 417-430 (2014)

23. L Liu et al., Physarum optimization: a biology-inspired algorithm for the Steiner tree problem in networks. IEEE Trans. Comput. 64(3), 819-832 (2015)

24. Y Liu et al., Multi-layer clustering routing algorithm for wireless vehicular sensor networks. IET Commun. 4(7), 810-816 (2010)

25. B Costas et al., Approximating congestion + dilation in networks via "Quality of Routing" games. IEEE Trans. Computers 61(9), 1270-1283 (2012)

26. T Meng et al., Spatial reusability-aware routing in multi-hop wireless networks. IEEE TC, 2015. doi:10.1109/TC.2015.2417543

27. A Dvir et al., Backpressure-based routing protocol for DTNs. ACM SIGCOMM Computer Communication Review 41(4), 405-406 (2011)

28. Y Yun-Sheng et al., Flooding-limited and multi-constrained QoS multicast routing based on the genetic algorithm for MANETs. Math. Comput. Model. 53(11-12), 2238-2250 (2011)

29. S Thrasyvoulos et al., Routing for disruption tolerant networks: taxonomy and design. Wirel. Netw 16(8), 2349-2370 (2010)

30. A Vasilakos et al., Delay tolerant networks: protocols and applications (CRC Press, Boca Raton, 2012)

31. I Woungang et al., Routing in Opportunistic Networks (Springer book, 2013), pp. 353-382. doi: 10.1007/978-1-4614-3514-3_13

32. Z Xin Ming et al., Interference-based topology control algorithm for delay-constrained mobile ad hoc networks. IEEE Trans. Mob. Comput. 14(4), 742-754 (2015)

33. PBF Duarte et al., "On the partially overlapped channel assignment on wireless mesh network backbone: A game theoretic approach", selected areas in communications. IEEE Journal on 30(1), 119-127 (2012)

34. A Alireza et al., A survey of security challenges in cognitive radio networks: solutions and future research directions. Proc. IEEE 100(12), 3172-3186 (2012)

35. AV Vasilakos et al., Information centric network: research challenges and opportunities. J. Netw. Comput. Appl. 52, 1-10 (2015)

36. S Marwaha et al., Evolutionary fuzzy multi-objective routing for wireless mobile ad hoc networks. Evol. Comput. 2, 1964-1971 (2004). CEC2004. Congress on

37. A Vasilakos et al., Optimizing QoS routing in hierarchical ATM networks using computational intelligence techniques. Systems, Man, and Cybernetics, Part C: Applications and Reviews, IEEE 33, 297 (2003)

38. W Quan et al., TB2F: tree-bitmap and bloom-filter for a scalable and efficient name lookup in content-centric networking. IFIP Networking, 2014

39. Y Yao et al., EDAL: An Energy-Efficient, Delay-Aware, and Lifetime-Balancing Data Collection Protocol for Wireless Sensor Networks IEEE/ACM TRANSACTIONS ON NETWORKING 23(3), pp. 182-190, (2013). doi: 10.1109/ MASS.2013.44

40. Y Yanjun et al., EDAL: An Energy-Efficient, Delay-Aware, and LifetimeBalancing Data Collection Protocol for Heterogeneous Wireless Sensor Networks. IEEE/ACM Trans. Networking 23(3), 810-823 (2015)

41. L Xiang et al., Compressed data aggregation for energy efficient wireless sensor networks, Proc. 8th, IEEE SECON, pp. 46-54, 2011, doi: 10.1109/SAHCN.2011. 5984932

42. N Chilamkurti et al., Cross-layer support for energy efficient routing in wireless sensor networks. Journal of Sensors 2009, 9 (2009)

43. X-Y Liu et al., CDC: compressive data collection for wireless sensor networks. IEEE Transactions on Parallel \& Distributed Systems 26(8), 2188-2197 (2015)

44. K Liu et al., A cooperative MAC protocol with rapid relay selection for wireless ad hoc networks. Comput. Netw. 91, 262-282 (2015)

45. Y Mao et al., Software-defined and virtualized future mobile and wireless networks: a survey. ACM/Springer Mobile Networks and Applications 20(1), 4-18 (2015)

46. W Xiaofei et al., A survey of green mobile networks: opportunities and challenges. MONET 17(1), 4-20 (2012)
47. A Keränen, J Ott, T Kärkkäinen, The ONE simulator for DTN protocol evaluation. Proceedings of the 2 nd International Conference on Simulation Tools and Techniques, 2009. doi:10.4108/ICST.SIMUTOOLS2009.5674

48. F Ekman, A Keränen, J Karvo, J Ott, Working day movement model. Proceedings of the 1st ACM SIGMOBILE workshop on Mobility models, 2008, pp. 33-40. doi:10.1145/1374688.1374695

\section{Submit your manuscript to a SpringerOpen ${ }^{\odot}$ journal and benefit from:}

- Convenient online submission

- Rigorous peer review

- Immediate publication on acceptance

- Open access: articles freely available online

- High visibility within the field

- Retaining the copyright to your article

Submit your next manuscript at springeropen.com 